\title{
Особенности циклотронного резонанса в трехмерных топологических изоляторах
}

\author{
(C) Р.В. Туркевич ${ }^{1,2}$, В.Я. Демиховский ${ }^{1}$, А.П. Протогенов ${ }^{1,2}$ \\ ${ }^{1}$ Нижегородский государственный университет, \\ 603950 Нижний Новгород, Россия \\ ${ }^{2}$ Институт прикладной физики Российской академии наук, \\ 603950 Нижний Новгород, Россия \\ E-mail: roman-turkevich@mail.ru
}

(Получена 27 апреля 2017 г. Принята к печати 12 мая 2017 г.)

Изучаются особенности циклотронного резонанса в топологических изоляторах семейства $\mathrm{Bi}_{2} \mathrm{Te}_{3}$. Энергетический спектр электронных возбуждений на поверхности этих соединений в магнитном поле обладает симметрией $C_{3 V}$. Используя квазиклассические уравнения движения, которые учитывают кривизну Берри и магнитный момент, мы нашли, что спектр циклотронных частот содержит дополнительные гармоники, связанные с анизотропией спектра. Исследование этих гармоник позволяет прояснить вклад топологических характеристик в наблюдаемые величины.

DOI: 10.21883/FTP.2017.11.45109.23

\section{1. Введение}

Исследования топологических изоляторов в течение последних десяти лет позволили прояснить многие фундаментальные свойства этих фазовых состояний. Последовавшие за открытием графена и продолженные изучением дираковских и вейлевских полуметаллов исследования различных модификаций топологических изоляторов позволили построить новый класс так называемых дираковских материалов. Одним из ключевых свойств этих сред является безмассовый релятивистский спектр низкоэнергетических поверхностных или краевых возбуждений. Другим свойством является существование глобальной симметрии, например симметрии относительно инверсии времени, которая защищает поверхностные и краевые состояния от рассеяния назад при умеренном структурном беспорядке. Фундаментальное отличие топологических изоляторов от стандартных зонных изоляторов определяется ненулевыми значениями топологических инвариантов. Описание перечисленных свойств топологических изоляторов отражено в известных обзорах $[1,2]$ и монографиях $[3,4,5]$.

Особенностью проявления эффектов, связанных с нетривиальной топологией, является коротковолновый характер корреляций блоховских электронных состояний. Он закодирован в фазе волновых функций электронов в форме фазы Берри и соответствующем ей числе Черна. Как правило, топологические поправки такого рода к наблюдаемым величинам малы и требуют использования сверхсильных магнитных полей. Проявление топологических эффектов в вейлевских полуметаллах связано с существованием киральной симметрии, что ведет к киральной аномалии [6]. В вейлевских полуметаллах суммарное число Черна оказывается равным нулю. Несмотря на отмеченные трудности в выявлении топологических эффектов, новая картина, возникающая в физике металлов, радикально отличается от той, с которой исследователи имели дело ранее. Одним из фрагментов прежней картины физических явлений были резонансные эффекты в одноэлектронных и коллективных задачах физики конденсированного состояния. В частности, исследование циклотронного резонанса при наложении внешнего магнитного поля было направлено на изучение ферми-поверхности в металлах.

Цель настоящей работы заключается в изучении эффектов, связанных с циклотронным резонансом в трехмерных топологических изоляторах, и их использование в качестве инструмента для исследования проявлений топологических особенностей электронных состояний в этих средах. Нетривиальное фазовое состояние трехмерного топологического изолятора определяется ненулевым значением главного из четырех $\mathbf{Z}_{2}$ инвариантов и обусловлено существованием симметрии относительно инверсии времени. Во внешнем магнитном поле эта симметрия нарушается, в спектре генерируется щель и состояние характеризуется кривизной Берри и ненулевым значением числа Черна. Поэтому в работе мы сосредоточимся на изучении проявления кривизны Берри в явлении циклотронного резонанса. При решении этой задачи мы будем использовать квазиклассические уравнения, содержащие кривизну Берри [7]. Эффекты, связанные с существованием кривизны Берри, были проанализированы в рамках нестандартной динамики электронов в трехмерных топологических изоляторах в работе [8] без анализа гармоник циклотронного резонанса. В настоящей работе мы обратимся к особенностям спектра в соединениях $\mathrm{Bi}_{2} \mathrm{Te}_{3}$, где наблюдается наиболее сильное гексагональное искажение спектра дираковских состояний. Это позволит выявить влияние топологических характеристик на гармоники циклотронного резонанса в наибольшей степени. 


\section{2. Модель и основные уравнения}

Топологические изоляторы семейства $\mathrm{Bi}_{2} \mathrm{Te}_{3}$ содержат поверхностные электронные состояния, которые мы будем описывать эффективным гамильтонианом $[9,10]$

$$
H=\frac{\hbar^{2} k^{2}}{2 m}+\hbar v\left(k_{x} \sigma_{y}-k_{y} \sigma_{x}\right)+\hbar \frac{\lambda}{2}\left(k_{+}^{3}+k_{-}^{3}\right) \sigma_{z}+\Delta \sigma_{z} .
$$

Здесь $m$ - эффективная масса, $v$ - характерная скорость, $\mathbf{k}$ - волновой вектор, $\sigma_{i}$ - матрицы Паули, $\lambda-$ параметр гексагонального искривления, $k_{ \pm}=k_{x} \pm i k_{y}$, $\Delta$ - параметр модели, который дает значение дираковской щели при наличии, например, внешнего магнитного поля.

Представление о динамике электронных состояний можно получить, рассматривая спектр гамильтониана (1), который в полярных координатах равен

$$
E_{0}(k, \theta)=\frac{\hbar^{2} k^{2}}{2 m}+\sqrt{(\hbar k v)^{2}+\left(\Delta+\lambda \hbar k^{3} \cos 3 \theta\right)^{2}} .
$$

Параметры $m$ и $\lambda$ в этом выражении описывают слабые отклонения [11] от линейной зависимости, описываемой первым основным слагаемым в подкоренном выражении (2). В дальнейшем мы опустим, для простоты, первое слагаемое в (2). Из выражения (2) видно, что модификация щели $\Delta(k)=\Delta+\lambda \hbar k^{3} \cos 3 \theta$ в спектре возбуждений связана с параметром $\lambda$ гексагонального искривления.

Рассмотрим ситуацию, когда извне наложены постоянное магнитное поле с напряженностью $\mathbf{B}$ и переменное электрическое поле с напряженностью $\mathbf{E}$. Уравнения движения для центра электронного волнового пакета в этом случае в квазиклассическом приближении имеют вид [7]

$$
\begin{gathered}
\dot{\mathbf{r}}=\frac{1}{\hbar} \nabla_{\mathbf{k}} E_{\mathbf{k}}-[\dot{\mathbf{k}} \times \mathbf{\Omega}], \\
\hbar \dot{\mathbf{k}}=-e \mathbf{E}-e[\dot{\mathbf{r}} \times \mathbf{B}] .
\end{gathered}
$$

В уравнениях (3) и (4) введены следующие обозначения: $e-$ заряд электрона, $E_{k}=E_{k}^{0}-m_{\mathbf{k}} \mathbf{B}, E_{k}^{0}-$ закон дисперсии электронов в отсутствие магнитного поля, $m_{\mathbf{k}}$ - магнитный момент, связанный с орбитальным вращением вокруг центра волнового пакета.

В выражении (3) $\Omega_{\mathbf{k}}=\left[\nabla_{\mathbf{k}} \times \mathbf{A}_{\mathbf{k}}\right]-$ кривизна Берри, где $\mathbf{A}_{\mathbf{k}}=i\left\langle u_{\mathbf{k}}\left|\nabla_{\mathbf{k}}\right| u_{\mathbf{k}}\right\rangle-$ связность Берри, а $u_{\mathbf{k}}-$ периодическая часть функции Блоха. Упомянутое выше число Черна равно $C=\frac{1}{2 \pi} \int \Omega_{z} d^{2} k$, так как в рассматриваемой нами динамике поверхностных состояний вектор $\Omega=\left(0,0, \Omega_{z}\right)$ направлен по нормали к поверхности топологического изолятора. Орбитальный магнитный момент равен $m_{\mathbf{k}}=-(e / 2 \hbar) \operatorname{Im}\left[\left\langle\nabla_{\mathbf{k}} u_{\mathbf{k}}\left|\mathscr{H}-E_{\mathbf{k}}^{0}\right| \nabla_{\mathbf{k}} u_{\mathbf{k}}\right\rangle\right]$. Выражение для магнитного момента в простейшем случае двух энергетических зон имеет особенно простой вид: $m_{\mathbf{k}}=-(e / 2 \hbar)\left(E_{\mathbf{k}}^{c}-E_{\mathbf{k}}^{v}\right) \Omega_{\mathbf{k}}^{c}$. Здесь $E_{\mathbf{k}}^{v}$ и $E_{\mathbf{k}}^{c}-$ энергия электронов в валентной зоне и в зоне проводимости. Орбитальный магнитный момент и кривизна Берри в рассматриваемой модели (1) топологического изолятора $\mathrm{Bi}_{2} \mathrm{Te}_{3}$ равны [11]:

$$
\begin{gathered}
m_{z}\left(k_{x}, k_{y}\right)=-\frac{e}{2 \hbar c} \\
\times \frac{\hbar^{2} v^{2}\left(\Delta-2 \lambda \hbar k_{x}^{3}+6 \lambda \hbar k_{x} k_{y}^{2}\right)}{\left(\hbar^{2} v^{2}\left(k_{x}^{2}+k_{y}^{2}\right)+\left(\Delta+\hbar \lambda\left(k_{x}^{3}-3 k_{x} k_{y}^{2}\right)\right)^{2}\right)}, \\
\Omega_{z}\left(k_{x}, k_{y}\right)=-\frac{\hbar^{2} v^{2}\left(\Delta-2 \lambda \hbar k_{x}^{3}+6 \lambda \hbar k_{x} k_{y}^{2}\right)}{2\left(\hbar^{2} v^{2}\left(k_{x}^{2}+k_{y}^{2}\right)+\left(\Delta+\hbar \lambda\left(k_{x}^{3}-3 k_{x} k_{y}^{2}\right)\right)^{2}\right)^{\frac{3}{2}}},
\end{gathered}
$$

Обратим внимание на то, что наложение внешнего магнитного поля нарушает симметрию относительно инверсии времени, а в присутствии гексагонального искажения нарушается пространственная инверсия. В результате соответствующие стандартные симметрийные ограничения на кривизну Берри: $\Omega(k)=-\Omega(-k)$, $\Omega(k)=\Omega(-k)$, как следует из формулы $(6)$, нарушаются.

\section{3. Гармоники циклотронной частоты}

Из уравнений движения (3) и (4) и формул (5), (6) видно, что скорость движения вдоль изоэнергетических линий не постоянна. Она уменьшается на участках с положительной кривизной и увеличивается на промежутках, где кривизна траектории отрицательна. Существование осцилляций скорости приводит к тому, что динамика электрона содержит циклотронные частоты, которые являются дополнительными по отношению к стандартной.

Рассмотрим состояния электронов с энергией, близкой к энергии Ферми. Для определения дополнительных частот изучим поведение электрона в постоянном магнитном и переменном электрическом полях. Пусть магнитное поле направлено перпендикулярно плоскости вращения, а переменное электрическое лежит в этой плоскости, являющейся поверхностью топологического изолятора. Зададим циркулярную поляризацию электрического поля следующим образом: $\varepsilon_{x}(t)=-\varepsilon_{0} \sin (\omega t)$, $\varepsilon_{y}(t)=\varepsilon_{0} \cos (\omega t)$. При таком выборе поляризации вращение вектора электрического поля будет происходить в том же направлении, в котором вращается электронное возбуждение. Чтобы идентифицировать резонансные гармоники, удобно отслеживать зависимость энергии от частоты электрического поля. Для определения энергии нам потребуется зависимость $\mathbf{k}(t)$. Из уравнений (3) и (4) следует, что система уравнений для определения этой зависимости имеет вид:

$$
\begin{aligned}
\hbar \dot{k}_{x} & =-\frac{1}{\left(1+\Omega_{z} / l_{b}^{2}\right)}\left(\frac{1}{l_{b}^{2}} \frac{\partial E_{k}}{\partial k_{y}}+e \varepsilon_{x}\right), \\
\hbar \dot{k}_{y} & =\frac{1}{\left(1+\Omega_{z} / l_{b}^{2}\right)}\left(\frac{1}{l_{b}^{2}} \frac{\partial E_{k}}{\partial k_{x}}-e \varepsilon_{y}\right) .
\end{aligned}
$$

Заметим, что в более общей геометрии, связанной со взаимной ориентацией полей, в правой части уравне- 


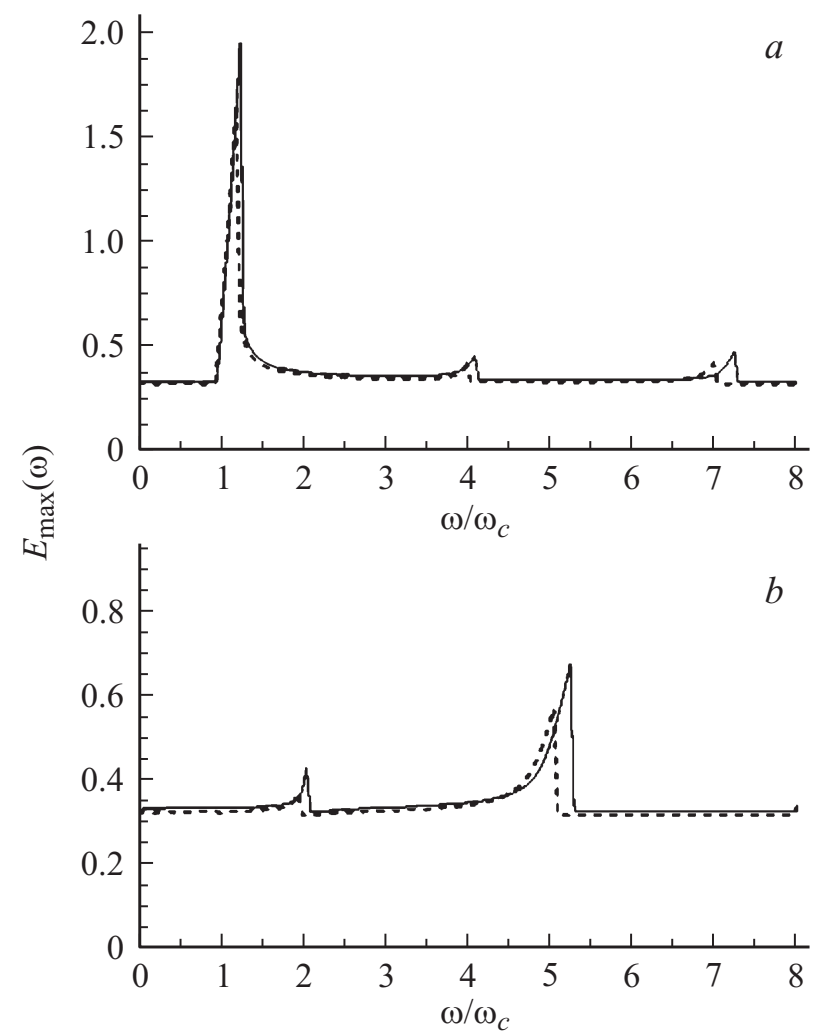

Зависимость максимально достигаемой энергии, измеряемой в эВ, от частоты электрического поля. Величина магнитного поля равна $10^{6}$ Гс. Пунктирная линия отвечает ситуации, когда магнитный момент и кривизна Берри не учитываются. Сплошная линия соответствует случаю, когда магнитный момент и кривизна Берри не равны нулю. Вектор электрического поля вращается в ту же сторону, что и электрон $(a)$, в противоположную сторону $(b)$ по отношению к направлению вращения электрона.

ний (7), (8) внутри скобок будет присутствовать слагаемое $e^{2}(\mathbf{E} \cdot \mathbf{B}) \Omega_{\mathbf{k}}$.

Для получения решения этой системы уравнений мы воспользуемся численными методами. Полученная зависимость энергии от времени показывает, что при некоторых частотах $\omega$ происходит существенное ее возрастание. В рассматриваемой системе резонанс является нелинейным, что объясняет конечный рост энергии электрона. Найдем максимально достигаемую энергию электрона как функцию частоты электрического поля. Эта зависимость показана на рисунке. Обычно в экспериментах по изучению циклотронного резонанса вместо энергии рассматривается поглощаемая мощность. Поэтому с экспериментальной точки зрения гармоники циклотронной частоты будут соответствовать максимумам поглощаемой мощности. Рассмотренные нами случаи $(a)$ и $(b)$ на рисунке отвечают различным круговым поляризациям электрического поля.

Аналитическое вычисление циклотронной частоты с учетом гексагонального искривления в $\mathrm{Bi}_{2} \mathrm{Te}_{3}$ затруднительно. Для оценки ее значения можно воспользоваться формулой для изотропного случая, когда $E(k)=\hbar v k$ :

$$
\omega_{c}^{\Omega}=\omega_{c} \frac{\left(1+2 \Omega_{z} / l_{b}^{2}\right)}{\left(1+\Omega_{z} / l_{b}^{2}\right)} .
$$

Здесь $\omega_{c}=e B / m_{c}$ и $m_{c}=E_{\mathrm{F}} / v^{2}$ - циклотронная масса. Формула (9) была получена в работе [8] без учета магнитного момента. Более общая формула, учитывающая магнитный момент (приводящая к отличию числителя в (9) от единицы), получена в работе [12]. В численных вычислениях циклотронной частоты с использованием уравнений движения (3) и (4) и выражения для спектра (2) значение для циклотронной частоты отличается от ее оценки (9) на $50 \%$.

Для пояснения появления в численных вычислениях частот, кратных циклотронной частоте $\omega_{c}^{\Omega}$, мы упрощенно представим решение уравнения (3) в виде гармоник

$$
r(t)=r_{0}+\sum_{n} a_{n} \cos \left(n \omega_{c}^{\Omega} t\right),
$$

где $r_{0}$ - определяет центр циклотронной орбиты, слагаемое $a_{n} \cos \left(n \omega_{c}^{\Omega} t\right)$ - описывает искажение круговой траектории и имеет ось симметрии $C_{n}, a_{n}-$ амплитуда искажения, $n-$ порядок оси симметрии. Совершая преобразование Фурье скорости $v_{x}(t)$, получим, опуская отрицательные частоты, что фурье-образ скорости имеет вид

$$
\begin{aligned}
F(\omega)= & \gamma_{1} \delta\left(\omega-\omega_{c}^{\Omega}\right)+\gamma_{2} \delta\left(\omega-(n-1) \omega_{c}^{\Omega}\right) \\
& +\gamma_{3} \delta\left(\omega-(n+1) \omega_{c}^{\Omega}\right),
\end{aligned}
$$

где $\gamma_{i}-$ константы. Из этого выражения видно, что частотная зависимость состоит из трех $\delta$-функций в точках $\omega_{c}^{\Omega},(n-1) \omega_{c}^{\Omega}$ и $(n+1) \omega_{c}^{\Omega}$. Отсюда следует, что в рассматриваемой модели может наблюдаться резонанс на этих частотах.

$\mathrm{B} \mathrm{Bi}_{2} \mathrm{Te}_{3}$ энергетический спектр в отсутствие магнитного поля обладает осью симметрии $C_{6}$. Во внешнем магнитном поле энергия, связанная с магнитным моментом, и кривизна Берри обладают осью симметрии $C_{3}$ (см. выражения (5) и (6), где $\Delta_{\mathrm{eff}}=\Delta / 2-\lambda \hbar k_{x}^{3}$ $\left.+3 \lambda \hbar k_{x} k_{y}^{2}=\Delta / 2+\lambda \hbar k^{3} \cos 3 \theta\right)$. Отсюда можно сделать вывод о том, что резонансные частоты $(6 \pm 1) \omega_{c}^{\Omega}$ возникают благодаря гексагональному искривлению спектра, а частоты $(3 \pm 1) \omega_{c}^{\Omega}$ появляются в результате учета слагаемых, пропорциональных $\lambda$ в выражениях (5) и (6).

\section{4. Обсуждение}

Отличные от нуля значения кривизны Берри и магнитного момента приводят к сдвигу $\omega_{c}^{\Omega}-\omega_{c}$ частоты $\omega_{c}$. Экспериментальные возможности для наблюдения этого явления в соединении $\mathrm{Bi}_{2} \mathrm{Te}_{3}$, где параметр гексагонального искажения $\lambda=3.2 \cdot 10^{-7} \mathrm{~cm}^{3} / \mathrm{c}$ максимален, являются ограниченными в силу ряда обстоятельств. 
Очевидное желание использовать сверхсильные магнитные поля наталкивается на ограничения, связанные с зонной структурой используемых соединений и со способом создания таких полей в экспериментальных установках. По этой причине мы выберем для оценок предельно возможное значение напряженности стационарного магнитного поля, равное $5 \cdot 10^{5}$ Гс. При таком выборе величины магнитного поля энергия циклотронного кванта равна $\hbar \omega_{c}=0.03$ эВ. Величина запрещенной зоны в $\mathrm{Bi}_{2} \mathrm{Te}_{3}$ приближенно равна 0.15 эВ, а энергия Ферми, отсчитанная от дираковской точки, равна 0.29 эВ. В результате под уровнем Ферми будут расположены несколько уровней Ландау. Поскольку топологическая информация содержится в фазе волновой функции, а не в гамильтониане, различия уравнений движения (4) в квазиклассическом пределе и при квантовом способе описания в случае, когда заполнено несколько уровней Ландау, не существенны для оценок величин рассматриваемых эффектов. В случае использования импульсного магнитного поля его величина может быть увеличена до $10^{6}$ Гс при применении неразрушающих техник.

Для реализации циклотронного резонанса необходимо, чтобы частота поля $\omega$, равная циклотронной частоте, при $m_{c}=0.3 m_{e}$, была больше плазменной частоты $\omega_{p}=\sqrt{4 \pi n e^{2} / \varepsilon m}$ в рассматриваемой системе [13]. При концентрации $n=10^{17} \mathrm{~cm}^{-3}$, скорости $v=3.8 \cdot 10^{7} \mathrm{~cm} / \mathrm{c}$ и $\varepsilon=58$ плазменная частота равна $\omega_{p}=2.3 \cdot 10^{12} \mathrm{c}^{-1}$. При частотах, меньших $\omega_{p}$, необходимо учитывать скинэффект. Отметим, что циклотронная частота в рассматриваемом случае магнитного поля с напряженностью $5 \cdot 10^{5}$ Гс равна $\omega_{c}=5.1 \cdot 10^{13} \mathrm{c}^{-1}$. Обратим внимание также на то, что плазменная частота в двумерной [13] дираковской плазме $\omega_{p}=\sqrt{r_{s}}(\pi n)^{1 / 4} v q^{1 / 2}, r_{s}=e^{2} /(\varepsilon v \hbar)$, уже при значении $q=1 \mathrm{~cm}^{-1}$ волнового вектора $q$ оказывается значительно меньше рассмотренных выше частот. В частности, при $n=6 \cdot 10^{17} \mathrm{~cm}^{-2}$ она равна $2.2 \cdot 10^{10} \mathrm{c}^{-1}$.

Использование в эксперименте гелиевых температур связано с необходимостью уменьшения размытия функции распределения электронов и с подавлением рассеяния их на фононах. Оценка обратного времени жизни электронных состояний при рассеянии на примесях в оптимальном случае дает значение $10^{-11} \mathrm{c}^{-1}$. В этих условиях относительная величина $\left(\omega_{c}^{\Omega}-\omega_{c}\right) / \omega_{c}$ сдвига частоты $\omega_{c}$ составляет $1 \%$. Поскольку гексагональное искажение спектра является сильным, амплитуда сигнала на дополнительных гармониках по отношению к основной, как мы ожидаем, будет находиться в пределах, доступных для экспериментальной проверки.

Дополнительная возможность обнаружения нетривиальных топологических особенностей отклика на внешнее поле связана с изучением генерации второй гармоники в рассматриваемых соединениях во внешнем магнитном поле. Дело в том, что в центре зоны Бриллюэна этих соединений над принципиальным конусом Дирака находится второй конус Дирака [14,15], принадлежащий незанятым состояниям. Его использование в теории [16] и в эксперименте [14] по двухфотонному поглощению указывает на еще один способ выявления вклада топологических характеристик в наблюдаемые величины.

\section{5. Заключение}

Резюмируя отметим, что при изучении явления циклотронного резонанса в трехмерных топологических изоляторах семейства $\mathrm{Bi}_{2} \mathrm{Te}_{3}$, имеющих гексагональную симметрию электронного спектра, мы обнаружили дополнительные резонансные гармоники, кратные основной. Особенность спектра дополнительных частот состояла в том, что в нем проявились частоты $(3 \pm 1) \omega_{c}^{\Omega}$. Причина их существования в энергетическом спектре состояний и в отклике на внешнее поле одна и та же. Она определяется структурой вида $\Delta_{\text {eff, }}$ которая присутствует в формулах (5) и (6). Это означает, что появление дополнительных гармоник можно использовать для идентификации топологически нетривиального состояния, связанного с присутствием фазы Берри в рассматриваемых соединениях.

Эта работа была поддержана проектом № 3.3026.2017/ПЧ Министерства Образования и Науки Российской Федерации (Р.В.Т.)

\section{Список литературы}

[1] M.Z. Hasan, C.L. Kane. Rev. Mod. Phys., 82, 3045 (2010).

[2] X.-L. Qi, S.-C. Zhang. Rev. Mod. Phys., 83, 1057 (2011).

[3] G.E. Volovik, The Universe in a Helium Droplet (Oxford University Press, N.Y., 2003).

[4] B.A. Bernevig (with T.L. Hughes). Topological insulators and topological superconductors (Princeton University Press, 2013).

[5] S.-Q. Shen. Topological insulators (Springer, 2012).

[6] S. Adler. Phys. Rev., 177, 2426 (1969).

[7] G. Sundaram, Q. Niu. Phys. Rev. B, 59, 14915 (1999).

[8] В.Я. Демиховский, Р.В. Туркевич. Письма ЖЭТФ, 101, 501 (2015).

[9] L. Fu. Phys. Rev. Lett., 103, 266801 (2009).

[10] M. Nomura, S. Souma, A. Takayama, T. Sato, T. Takahashi, K. Eto, Kouji Segawa, Yoichi Ando. Phys. Rev. B, 89, 045134 (2014).

[11] Z. Li, J.P. Carbotte. Phys. Rev. B, 89, 165420 (2014).

[12] Francesco M.D. Pellegrino, Mikhail I. Katsnelson, Marco Polini. Phys. Rev. B, 92, 201407 (2015).

[13] S. Das Sarma, E.H. Hwang. Phys. Rev. Lett., 102, 206412 (2009).

[14] D. Niesner, Th. Fauster, S.V. Eremeev, T.V. Menshchikova, Y.M. Koroteev, A.P. Protogenov, E. V. Chulkov, O.E. Tereshchenko, K.A. Kokh, O. Alekperov, A. Nadjafov, N. Mamedov. Phys. Rev. B, 86, 205403 (2012).

[15] S.V. Eremeev, I.V. Silkin, T.V. Menshchikova, A.P. Protogenov, E.V. Chulkov. JETP Lett., 96, 780 (2013).

[16] Takahiro Morimoto, Shudan Zhong, Joseph Orenstein, Joel E. Moore. arXiv:1609.05932.

Редактор А.Н. Смирнов 


\section{Cyclotron resonance features}

in a three-dimensional topological

insulators

R.V. Turkevich ${ }^{\mathbf{1}, 2}$, V.Ya. Demikhovskii $^{\mathbf{1}}$, A.P. Protogenov ${ }^{1,2}$

${ }^{1}$ Nizhny Novgorod State University, 603950 Nizhny Novgorod, Russia

${ }^{2}$ Institute of Applied Physics

of Russian Academy of Scienes,

603950 Nizhny Novgorod, Russia

Abstract Cyclotron resonance features in topological insulators of the $\mathrm{Bi}_{2} \mathrm{Te}_{3}$ family are studied. The energy spectrum of electron excitations on the surface of these compounds in a magnetic field has $C_{3 V}$ symmetry. Using quasiclassical equations of motion, which take into account the Berry curvature and the magnetic moment, we have found that the spectrum of cyclotron frequencies has additional harmonics associated with anisotropy of the spectrum. Study of these harmonics allows determining the contribution of topological characteristics to observables. 